\title{
Self-Medication of Anti-Biotics amongst University Students of Islamabad: Prevalence, Knowledge and Attitudes
}

\author{
Muhammad Paras Javed \\ Department of Pharmaceutical Sciences, Riphah International University, Pakistan.
}

\begin{abstract}
The prevalence and pattern of self-medication with antibiotics among undergraduate and graduate community of students at different universities of Islamabad was evaluated using structured self-medication administered questionnaire. This cross-sectional, study was conducted in March 2013. A convenience sample was taken from 4 non-medical universities of the city of Islamabad, Pakistan. Data was analyzed using SPSS $v 14$ and associations were tested using the Chi square test. A total of 210 questionnaires were randomly distributed with a respondent rate of 100\%. The prevalence of self-medication was found to be $77.03 \%$ (Female: Male Ratio=1:1.14). The major reasons given for self-medicating with antibiotics were; $33.63 \%$ assumed knowledge on antibiotics $(P=0.478), 26.64 \%$ prior experience on use $(P=0.378)$, while $9.17 \%$ admitted lack of time to go for consultation $(P=0.130)$. Majority of respondents however, self-medicate with antibiotics occasionally. The most reported antibiotic class (48.58\%) was the $\beta$-lactams (as amoxicillin) while cotrimoxazole was rarely used (2.23\%). The most reported condition for self-medication was respiratory tract infections (59\%). About almost half of the respondents (46.79\%) purchased the drugs from drug stores. These findings highlight the needs for planning interventions to promote the judicious use of antibiotics within the student population.
\end{abstract}

Keywords: Antibiotics, drugs, Pakistan, rational use, self-medication, students.

\section{Introduction}

Medication is the act of consuming medicines for diagnosis, prevention or treatment of diseases. Correct medication is observed by reporting any symptom or disease to the physician in the hospital or clinic who diagnoses and prescribes the needed drugs to alleviate the condition ${ }^{[1]}$.

Self medication can be defined as obtaining and consuming medication without professional supervision, which comprises of acquiring medicines without a prescription, purchasing drugs by resubmitting/reutilizing an old prescription, taking medicines on advice of relative or others, or consuming leftover medicines already available at home ${ }^{[2]}$. There is much public and professional concern about the irrational use of drugs ${ }^{[3]}$. The prevalence rates are high all over the world; in developing countries it is as high as $92 \%{ }^{[4]}$, up to $68 \%$ in European countries ${ }^{[5]}$. Our neighboring countries have a prevalence rate of $31 \%$ in India and $59 \%$ in Nepal ${ }^{[4]}$. Very few studies regarding self medication have been conducted in Pakistan which has also confirmed high rates of prevalence of around $51 \%{ }^{[6]}$.

The misuse of non-prescription drugs amongst students has become a serious problem. The youth is very much exposed to the media and the increased advertising of pharmaceutical products through electronic and print media pose a larger threat to the young population. This increases concerns of incorrect self-diagnosis, drug-drug interaction and use other than for the true indication. To our knowledge, studies have been done in Pakistan to assess self medication in the young generation.

A major problem with self-medication with antibiotics is the resistance of human pathogens. Selfmedication with antibiotics is of serious medical concern. Antibiotics resistance is a current problem world-wide particularly in developing countries, where antibiotics are often available without a prescription ${ }^{[7]}$. This study presents the results of the Pakistani youth's knowledge, attitude and practice towards self medication.

\section{Study Background}

The objective of this study was to evaluate the prevalence of self-medication among undergraduate and graduate students in Islamabad. A community based cross-sectional survey was conducted in different universities of Islamabad.

\section{Methodology}

Self administered, structured questionnaires were designed for the purpose of data collection. The questionnaires consisted of both closed and sometimes open-ended questions. The information on the questionnaires included social demographic variables such as gender, age and marital status. In addition to 
questions on demographic information, the questionnaire included questions on involvement in self-medication practices, frequency of self-medication, sources of antibiotics used, names of antibiotics used, reasons for selfprescribing of antibiotics, self-medication of antibiotics as a precautionary measure, and recommendation of physician for culture test. Data was analyzed using SPSS v14 and associations were tested using the Chi square test.

\section{Results}

The prevalence of self-medication was 77.03\% (161/209), out of which most of the respondents (63.97\%) reported that they get involved in self medication "occasionally", 18.63\% admitted that they selfmedicate "most of the time" while the remaining respondents claimed to self medicate "frequently" as seen in TABLE 1. Majority of the students $95(\mathrm{P}=0.540)$ reported that they self medicated for respiratory tract infections (RTIs) and skin infections (TABLE 2).

Table 1: Frequency of self-medication

\begin{tabular}{|l|l|l|}
\hline Response & Frequency & Percentage (\%) \\
\hline Occasionally & 103 & 63.97 \\
\hline Frequently & 28 & 17.40 \\
\hline Most of the time & 30 & 18.63 \\
\hline
\end{tabular}

Table 2: Conditions for which persons self-medicated

\begin{tabular}{|l|l|l|}
\hline Condition & Frequency & Probability* \\
\hline G.I.T Infections & 20 & 0.124 \\
\hline Genitourinary Infections & 10 & 0.062 \\
\hline Respiratory Tract Infections & 115 & 0.714 \\
\hline Skin Infections & 35 & 0.217 \\
\hline Others & 15 & 0.093 \\
\hline
\end{tabular}

*Multiple responses, total value doesn't add to 1 .

Table 3: Factors influencing self-medication

\begin{tabular}{|l|l|l|}
\hline Reasons for SM & Frequency & Probability* \\
\hline Knowledge about drugs & 77 & 0.478 \\
\hline Prior experience on use & 61 & 0.378 \\
\hline Lack of time & 21 & 0.130 \\
\hline Financial constraints & 11 & 0.068 \\
\hline Nonchalant attitude of clinic staff & 0.292 \\
\hline Illness considered not serious for consultation & 47 & 0.037 \\
\hline Others & 6 & 0.037 \\
\hline
\end{tabular}

*Multiple responses, total doesn't add to 1.

Table 4: Type of antibiotics used in self-medication

\begin{tabular}{|l|l|l|}
\hline Antibiotics & Frequency & Probability* \\
\hline Amoxicillin & 87 & 0.540 \\
\hline Tetracycline & 14 & 0.087 \\
\hline Ciprofloxacin & 21 & 0.130 \\
\hline Co-trimoxazole & 4 & 0.024 \\
\hline Cefuroxime & 5 & 0.031 \\
\hline Metronidazole & 12 & 0.074 \\
\hline Others & 34 & 0.211 \\
\hline
\end{tabular}

*Multiple responses, total doesn't add to 1.

Table 5: Source of purchase of antibiotics

\begin{tabular}{|l|l|l|}
\hline Place & Frequency & Probability* \\
\hline Hospital Pharmacy & 45 & 0.279 \\
\hline Community Pharmacy & 15 & 0.093 \\
\hline Private Clinic & 31 & 0.192 \\
\hline Drug store & 80 & 0.446 \\
\hline
\end{tabular}

*Multiple responses, total doesn't add to 1 . 
Table 6: Experience of persons about self-medication effectiveness

\begin{tabular}{|l|l|l|}
\hline Response & Male (\%) & Female (\%) \\
\hline Yes & 40.69 & 22.66 \\
\hline Sometimes & 39.55 & 66.67 \\
\hline No & 19.76 & 10.67 \\
\hline
\end{tabular}

The major reasons given for self medication included assumed knowledge on antibiotics $(\mathrm{P}=0.478)$ and prior experience on use $(\mathrm{P}=0.378)$. The most common antibiotic that was reported for self-medication was Amoxicillin with frequency of $87(\mathrm{P}=0.540)$ as indicated in TABLE 4. It was also observed that minor community of students take paracetamol and non-steroidal anti-inflammatory drugs (NSAIDs) instead of antibiotics in case of respiratory tract infections and also in gastro-intestinal infections.

Eighty $(\mathrm{P}=0.446)$ of the respondents purchased drugs directly from drug stores (private pharmacies) as given in Table 5. Other sources of the drugs included hospital pharmacies $45(\mathrm{P}=0.279)$, private clinics 31 $(\mathrm{P}=0.192)$ and community pharmacies $15(\mathrm{P}=0.093) .52 .17 \%$ of the student community reported the effectiveness of self-medication "sometimes". It has also been observed that only $33.54 \%$ of the students take antibiotic as precautionary measure. $60.86 \%$ had heard about antibiotic-resistant bacteria.

\section{Discussion}

The occurrence of antibiotic self-medication among undergraduate and graduate students is considerably high. The major factors associated with self medication with antibiotics is assumed knowledge on diseases and their treatments, prior experience on use of antibiotics and nonchalant attitude of hospital/clinical staff.

From this study, self medication with antibiotics was found to be dominantly related to assumed knowledge of antibiotics (TABLE 3). Although these students admitted to have knowledge on antibiotics, consequences of their misuse and hazards associated with self medication but alarmingly, these didn't deter them from indulging in this practice.

This practice is based upon the assumption that same or similar symptoms of a disease or infection could be treated with same drug and regimen as used earlier. These drugs may have been prescribed initially but they keep purchasing the same drugs or just use up the "left-overs" whenever the symptoms reappear. A number of students claimed that the negative attitude of some clinical staff kept them away from the clinic/hospital and so, indulge in self medication with antibiotics when they needed medication.

The disease conditions for which the students self-medicated are common health problems among many populations and this suggests that the trend of antibiotic self-medication may continue if proper measures are not taken. Amoxicillin was reported as the main drug used to self-medicate in this study (TABLE 4) and this correlates with the "assumed knowledge" of drugs as amoxicillin is probably used here because of its broad spectrum action to treat respiratory tract infections which is the major disease for which these students self medicated.

A major problem of self-medication with antibiotics is the emergence of drug resistance. Antimicrobial resistance is a current problem world-wide; particularly in developing countries. Inadequate dosing, incomplete treatment courses and indiscriminate drug use have major contributions to the emergence and progress of drug resistance in microbes. The consequence of this is the loss of relatively affordable drugs that will require new drugs development, which will be more expensive and will further put developing and progressing countries at disadvantages. The rational use of antibiotics is thus of utmost importance to limit the increase in bacterial resistance.

From the study, the major source of purchase of antibiotics was drug stores (private pharmacies) as mentioned in TABLE 5. Though regulations that categorize most of these drugs as prescription only exist, regulatory authorities and departments often lack resources to enforce them. Even if enforcement was possible, having and enforcing a strict prescription policy without providing adequate and affordable access to medical consultation and treatment, might exclude the lower class people from accessing drugs, leading to increased morbidity from otherwise curable infectious diseases.

\section{Conclusion}

The results from this study are limited to self-reported data, and as with all self-reported data, it depends on information given by the respondents. This study shows that self medication with antibiotics is widely common and inappropriate among university students. We recommend that a holistic approach must be taken to prevent this problem from escalating which would involve (i) Awareness and education regarding the 
implications of self-medication (ii) Strategies to prevent the supply of medicines without prescription by pharmacies (iii) Strict rules regarding pharmaceutical advertising and (iv) Policies to make receiving health care much less difficult. This study has also opened gateways for further research in this issue, besides showing that it is a real problem and should not be ignored.

\section{References}

[1] Olayemi O.J., Olayinka B.O., Musa A.I., Evaluation of Antibiotic Self-Medication pattern amongst undergraduate students of Ahmadu Bello University (Main Campus), Zaria. Russian Journal of Applied Sciences Engineering and Technology 2(1): 35-38 2010.

[2] Zafar SN, Reema S, Sana W, Akbar JZ, Talha V, Mahrine S, Wajeeha Y, Saman S, Sarah S. Self medication amongst university students of Karachi: prevalence, knowledge andattitudes. J Pak Med Assoc 2008, 58, 214-17.

[3] Filho L, Antonio I, Lima-Costa MF, Uchoa E. Bambui Project: a qualitative approach to self-medication. Cad Saude Publica 2004; 20: 1661-9.

[4] Shankar PR, Partha P, Shenoy N. Self-medication and non-doctor prescription practices in Pokhara valley, Western Nepal: a questionnaire-based study. BMC Fam Pract 2002; 3: 17.

[5] Bretagne JF, Richard Molyoivd B, Honnorat C, Caekaert A, Barthelemy P. [Gastroesophageal reflux in the French general population: national survey of 8000 adults]. Presse Med 2006; 35: 23-31.

[6] Haider S, Thaver IH, Self medication or self care: implication for primary health care strategies. J Pak Med Assoc 1995; 45: 297-8.

[7] Chalker, J., Improving antibiotic prescribing in Hai Phong Province, Viet Nam: he "antibiotic-dose" indicator. Bulletin of the World Health Organization, 2001; 79(4). 